\title{
Circular RNAs promote TRPM3 expression by inhibiting hsa-miR- $130 a-3 p$ in coronary artery disease patients
}

\author{
Ren-You Pan ${ }^{1,}{ }^{*}$, Ping Liu ${ }^{1}$, Hai-Tang Zhou ${ }^{1}$, Wei-Xin Sun ${ }^{1}$, Jun Song ${ }^{1, *}$, Jiang Shu ${ }^{1}$, \\ Guo-Jing Cui ${ }^{1}$, Zhi-Jian Yang ${ }^{2}$ and En-Zhi Jia ${ }^{2}$ \\ ${ }^{1}$ Department of Cardiovascular Medicine, Yancheng TCM Hospital Affiliated to Nanjing University of Chinese Medicine, \\ Yancheng 224000, Jiangsu Province, China \\ ${ }^{2}$ Department of Cardiovascular Medicine, The First Affiliated Hospital of Nanjing Medical University, Nanjing 210029, Jiangsu \\ Province, China \\ *These authors contributed equally to this work
}

Correspondence to: En-Zhi Jia, email: enzhijia@njmu.edu.cn

Keywords: microRNA, coronary heart disease, circRNA, ceRNA

Received: May 04, 2017 Accepted: July 25, $2017 \quad$ Published: August 04, 2017

Copyright: Pan et al. This is an open-access article distributed under the terms of the Creative Commons Attribution License 3.0 (CC BY 3.0), which permits unrestricted use, distribution, and reproduction in any medium, provided the original author and source are credited.

\section{ABSTRACT}

We investigated the differential expression of circular RNAs (circRNAs) in plasma samples from three coronary artery disease (CAD) patients to identify putative therapeutic targets. We identified 24 differentially expressed circRNAs (18 up-regulated and 6 down-regulated) and 7 differentially expressed mRNAs ( 6 upregulated and 1 down-regulated) in CAD patients based on competing endogenous RNA (ceRNA) microarray analysis. MiR-221 $(p=0.001), \operatorname{miR}-155(p=0.049)$, and miR-130a $(p=0.001)$ were downregulated in CAD patients based on qRT-PCR analysis of another independent population of 932 study subjects (648 CAD subjects and 284 controls). We constructed a hsa-miR-130a-3p-mediated circRNA-mRNA ceRNA network using the miRanda database. This included 9 circRNAs (hsa_circ_0089378, hsa_circ_0083357, hsa_circ_0082824, hsa_circ_0068942, hsa_circ_0057576, hsa_ circ_0054537, hsa_circ_0051172, hsa_circ_0032970, and hsa_circ_0006323) and 1 mRNA (transient receptor potential cation channel subfamily M member 3 [TRPM3]). We have shown that 9 circRNAs promote TRPM3 expression by inhibiting hsa-miR$130 a-3 p$ in CAD patients.

\section{INTRODUCTION}

Cardiovascular disease (CVD) is the leading cause of human morbidity and mortality worldwide. According to World Health Organization (WHO), approximately 17.5 million people die annually from CVD [1]. This represents $31 \%$ of all global deaths. Stroke and coronary artery disease (CAD) are the major causes of CVD related deaths [1]. In China, CVD accounts for 300 deaths out of every 100,000 individuals [2], while, nearly a quarter of the Western population suffers from CAD and stroke [3-4]. Therefore, it is essential to identify risk factors associated with CVD that could be used for diagnosis and treatment at an early stage. The role of non-coding RNAs (ncRNAs) has been recognized in various human pathologies and they represent great potential as $\mathrm{CAD}$ biomarkers.
Human genome sequencing shows that although only $3 \%$ of the human genome codes for proteins, $80 \%$ of the human genome are transcribed [5]. The numbers of non-coding transcripts greatly exceed protein-coding mRNAs and represent species complexity [6-7]. Long noncoding RNAs (lncRNAs) are noncoding RNAs that are longer than 200 nucleotides that regulate diverse cellular functions. Recently, IncRNAs have been detected in human plasma, which can be used as disease biomarkers [8]. Aberrant levels of lncRNAs have also been reported in plasma from CAD patients [9]. Circular RNAs (circRNAs) are another class of non-coding RNAs that regulate gene expression in eukaryotes [10]. The circRNAs are competing endogenous RNAs (ceRNAs) that act as a sponge for microRNAs (miRNAs) by complementary base paring and therefore regulate gene transcription [11-12]. 
However, the expression and biological functions of ceRNAs in CAD are unknown. Therefore, in the present study, we generated plasma ceRNA expression profiles of three pairs of CAD and control samples to investigate their potential role as diagnostic markers in CAD.

\section{RESULTS}

\section{Differentially expressed plasma circRNAs and mRNAs in CAD patients}

The circRNA expression profiles in human CAD and control plasma were compared by the scatter plot (Figure 1) and volcano plot filtering (Figure 2) to identify differentially expressed circRNAs. We identified 24 differentially expressed circRNAs (fold change $\geq 1.5$ and $P<0.05$ ) between CAD and control plasma (Table 1). Figure 3 shows the heat map of these 24 differentially expressed circRNAs. Among these, 18 circRNAs were up-regulated and 6 circRNAs were down-regulated in CAD plasma. Among the up-regulated circRNAs, 17 were exonic, and 1 intragenic. Among the downregulated circRNAs, 6 were exonic and 1 intronic. The circRNA, hsa_circ_0051686 was both intronic and exonic. SBC Human $(4 * 180 \mathrm{~K})$ ceRNA microarray profiling showed 6 up-regulated and 1 down-regulated mRNAs (fold change $\geq 1.5$ and $P<0.05$ ) in CAD patients (Table 2).

\section{Differentially expressed miRNAs in CAD}

To establish a circRNA-miRNA-mRNA ceRNA network, we compared plasma miRNA expression profiles of CAD and control subjects in another independent population that was previously reported [13]. We identified 9 CAD-related miRNAs including miR-122, miR-133b, miR-214, miR-21, miR-106a, miR-130a, miR-155, miR-221, and miR-125b. Among these, $\operatorname{miR}-221(p=0.001)$, miR-155 $(p=0.049)$, and miR-130a $(p=0.001)$ were downregulated in CAD subjects than in non-CAD subjects (Table 3).

\section{MicroRNA-mRNA interaction}

The hsa-miR-221-3p, hsa-miR-155-5p, and hsamiR-130a-3p were selected for integrated analysis of miRNA and mRNA profiling data. The miRNA target predictions were based on miRanda (release August 2010; http://www.microrna.org/). We performed integrated analysis of the inverse relations of expressed miRNAs and mRNAs in conjunction with their predicted targets and identified transient receptor potential cation channel subfamily M member 3 (TRPM3) as a target gene for hsamiR-130a-3p.

\section{CircRNA-microRNA interactions}

Recently, circRNAs have been identified as miRNA sponges that regulate gene expression. Therefore, we used miRanda database to investigate potential miRNAs that bind to circRNAs in CAD patients. We observed that hsamiR-221-3p, hsa-miR-155-5p, and hsa-miR-130a-3p were bound by 10,12, and 9 circRNAs, respectively (Table 4).

\section{Construction of ceRNA network}

Since circRNAs interact with miRNAs through miRNA response elements (MREs), we searched for putative hsa-miR-130a-3p MREs in the circRNAs using miRanda. We identified 9 circRNAs that had hsamiR-130a-3p binding sites and negatively associated with hsa-miR-130a-3p. These included hsa_circ_0089378, hsa_circ_0083357, hsa_circ_0082824, hsa_circ_0068942, hsa_circ_0057576, hsa_circ_0054537, hsa_circ_0051172, hsa_circ_0032970, and hsa_circ_0006323. Based on these data, we constructed a hsa-miR-130a-3p-mediated circRNA-mRNA ceRNA network with 9 circRNAs and 1 mRNA (Figure 4 and Table 5).

\section{DISCUSSION}

The ceRNAs play a key role in post-transcriptional regulation and have been implicated in cardiovascular disease [12, 14-15]. In our previous study, we reported several miRNAs associated with CAD, but their actions were unknown [13]. In order to deciphere the ceRNA mechanisms related to $\mathrm{CAD}$, we constructed a global ceRNA-miRNA-mRNA triple network using the miRanda database. We identified 9 circRNAs and 1 mRNA that formed a network with hsa-miR-130a-3p. This study sheds new insights to exploring the complex posttranscriptional regulatory networks via ceRNA interactions and identifying pathways that are altered in CAD. Subsequently, specific ceRNAs can be therapeutically used to modulate specific pathways that are involved in CAD pathology.

The miR-130 precursor is a small non-coding RNA that has been identified in mice (MI0000156, MI0000408), humans (MI0000448, MI0000748) and a range of vertebrate species (MIPF0000034). Mature miR-130 is generated by the dicer enzyme upon excision from the $3^{\prime}$ arm of the hairpin. MiR-130a-3p is inversely associated with coronary atherosclerosis with its down-regulation contributing to endothelial progenitor cell dysfunction in subjects suffering from coronary artery disease [16-18]. Therefore, miR-130a-3p has therapeutic potential for the prevention and treatment of CAD. Its regulation by ceRNAs as shown in this study represents one possible mode of regulation that is clinically applicable. 
Table 1: List of deregulated cirRNAs in 3 CAD patients

\begin{tabular}{|c|c|c|c|c|c|c|}
\hline circRNAs & $p$-value & Fold change (CAD/Control) & Regulation & circRNA_ID & chromosome & gene_symbols \\
\hline hsa_circ_0082169 & 0.039772045 & 4.122889654 & down & hsa_circ_0082169 & $\operatorname{chr} 7$ & RBM28 \\
\hline hsa_circ_0063721 & 0.045825944 & 1.298228361 & down & hsa_circ_0063721 & $\operatorname{chr} 22$ & KIAA0930 \\
\hline hsa_circ_0033974 & 0.013049935 & 1.958659858 & up & hsa_circ_0033974 & $\operatorname{chr} 14$ & None \\
\hline hsa_circ_0026666 & 0.031019957 & 1.79233974 & up & hsa_circ_0026666 & $\operatorname{chr} 12$ & MAP3K 12 \\
\hline hsa_circ_0039001 & 0.018213071 & 2.365089746 & up & hsa_circ_0039001 & $\operatorname{chr} 16$ & PPP4C \\
\hline hsa_circ_0027323 & 0.036944716 & 2.581094502 & up & hsa_circ_0027323 & $\operatorname{chr} 12$ & CDK4 \\
\hline hsa_circ_0083357 & 0.008380919 & 1.920146911 & up & hsa_circ_0083357 & chr8 & CTSB \\
\hline hsa_circ_0038998 & 0.028026625 & 1.645027714 & up & hsa_circ_0038998 & $\operatorname{chr} 16$ & PPP4C \\
\hline hsa_circ_0051686 & 0.046998952 & 2.148530811 & down & hsa_circ_0051686 & $\operatorname{chr} 19$ & MEIS3 \\
\hline hsa_circ_0089378 & 0.046800946 & 2.205308262 & up & hsa_circ_0089378 & $\operatorname{chr} 9$ & VAV2 \\
\hline hsa_circ_0032970 & 0.001427116 & 2.071558488 & up & hsa_circ_0032970 & $\operatorname{chr} 14$ & $\mathrm{TC} 2 \mathrm{~N}$ \\
\hline hsa_circ_0037340 & 0.012987448 & 2.053372933 & up & hsa_circ_0037340 & $\operatorname{chr} 16$ & EME2 \\
\hline hsa_circ_0068942 & 0.028198329 & 2.046034622 & up & hsa_circ_0068942 & $\operatorname{chr} 4$ & ADD1 \\
\hline hsa_circ_0080259 & 0.018189514 & 1.831242935 & up & hsa_circ_0080259 & chr7 & PSPH \\
\hline hsa_circ_0022839 & 0.034785158 & 2.41623517 & up & hsa_circ_0022839 & chr11 & SSSCA1 \\
\hline hsa_circ_0045491 & 0.008242488 & 4.027899063 & down & hsa_circ_0045491 & $\operatorname{chr} 17$ & ARSG \\
\hline hsa_circ_0051172 & 0.030127874 & 2.797573948 & up & hsa_circ_0051172 & $\operatorname{chr} 19$ & AXL \\
\hline hsa_circ_0059349 & 0.048466294 & 2.447186366 & down & hsa_circ_0059349 & $\operatorname{chr} 20$ & PRNP \\
\hline hsa_circ_0054537 & 0.014938614 & 1.983846543 & up & hsa_circ_0054537 & $\operatorname{chr} 2$ & PSME4 \\
\hline hsa_circ_0006323 & 0.047365052 & 3.494456249 & up & hsa_circ_0006323 & $\operatorname{chr} 1$ & DPYD \\
\hline hsa_circ_0082824 & 0.025375501 & 2.440902767 & up & hsa_circ_0082824 & $\operatorname{chr} 7$ & CUL1 \\
\hline hsa_circ_0028926 & 0.043763361 & 1.594160744 & down & hsa_circ_0028926 & $\operatorname{chr} 12$ & ACADS \\
\hline hsa_circ_0053278 & 0.021506782 & 2.140743547 & up & hsa_circ_0053278 & $\operatorname{chr} 2$ & IFT172 \\
\hline hsa_circ_0057576 & 0.020268992 & 3.322760286 & up & hsa_circ_0057576 & $\operatorname{chr} 2$ & HECW2 \\
\hline
\end{tabular}

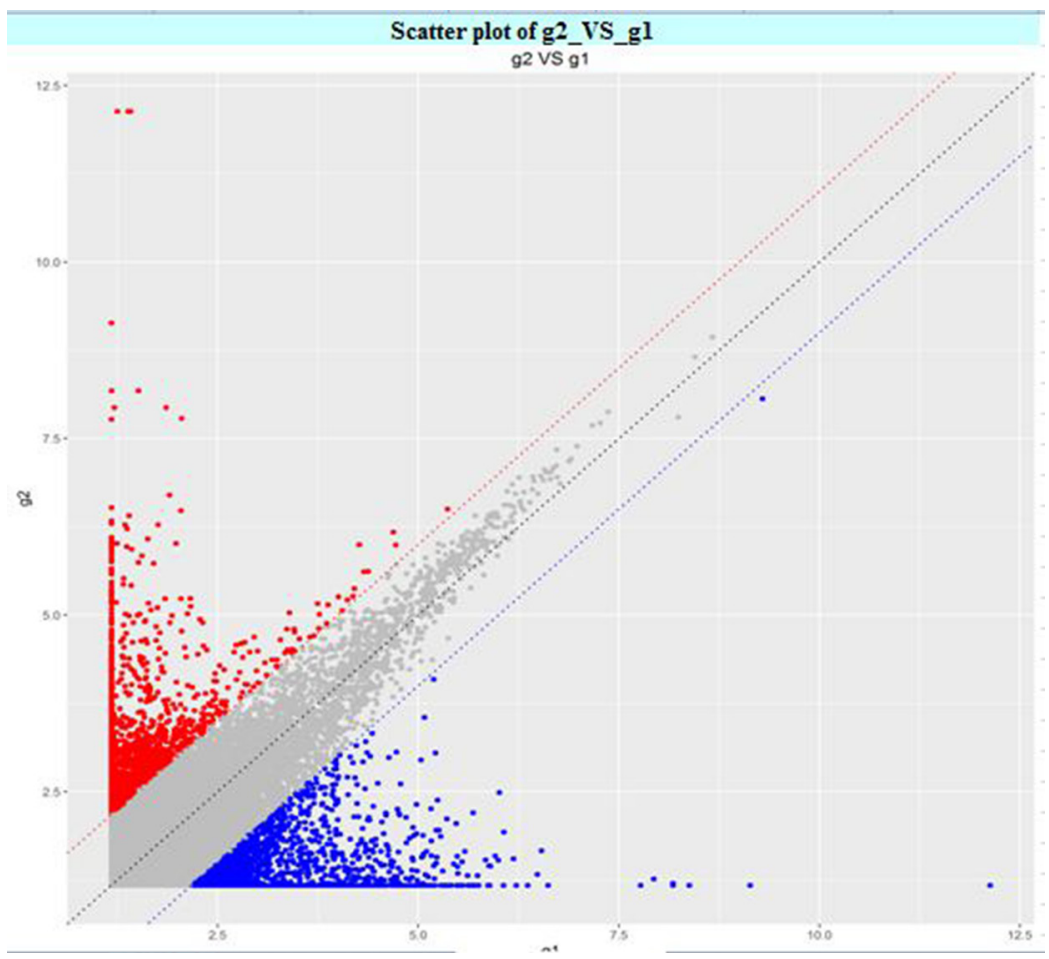

Figure 1: Scatter plot of differentially expressed plasma circRNAs in CAD and control subjects. The $X$ and $Y$ axis represent average signal values ( $\log 2$ scale) of plasma samples from CAD and control subjects. 
Table 2: List of deregulated mRNAs in 3 CAD patients

\begin{tabular}{ccccccc}
\hline \multicolumn{1}{c}{ mRNAs } & $\boldsymbol{p}$-value & $\begin{array}{c}\text { Fold change } \\
\text { (CAD/Control) }\end{array}$ & Regulation & Accession & Source & chr \\
\hline LNCV6_130905_PI430048170 & 0.030290601 & 1.886938464 & up & NM_000407 & RefSeq & chr22 \\
LNCV6_136795_PI430048170 & 0.041713357 & 2.873491196 & up & NM_078471 & RefSeq & chr17 \\
LNCV6_104643_PI430048170 & 0.005310873 & 2.792598516 & up & NM_001007471 & RefSeq & chr9 \\
LNCV6_141962_PI430048170 & 0.043928362 & 1.590466991 & up & NM_015136 & RefSeq & chr3 \\
LNCV6_98175_PI430048170 & 0.005752999 & 1.65921765 & up & NM_007129 & RefSeq & chr13 \\
LNCV6_128876_PI430048170 & 0.040154272 & 1.743659752 & up & NM_001270422 & RefSeq & chr17 \\
LNCV6_127855_PI430048170 & 0.022046703 & 2.447990837 & down & NM_001289088 & RefSeq & chr1 \\
\hline
\end{tabular}

TRPM3 belongs to the family of transient receptor potential (TRP) channels that regulate cellular calcium homeostasis. TRPM3 mediates calcium entry potentiated by calcium store depletion. Alternatively spliced transcript variants encoding different isoforms of TRPM3 have also been identified. TRPM3 regulates proliferation and contractility of vascular smooth muscle cells in co-ordination with cholesterol and is potentially involved in therapeutic vascular modulation [19]. In the present study, we identified TRPM3 mRNA as a hsa-miR-130a-3p target and is upregulated in CAD subjects.

Circular RNAs (circRNAs) are new members of ceRNAs that are involved in regulating gene expression [20].
However, their role in CAD pathogenesis has not been reported. In the present study, we identified 24 aberrantly expressed circRNAs (18 up-regulated and 6 down-regulated) in CAD patients. These included 9 circRNAs,namely, hsa_circ_0089378, hsa_circ_0083357, hsa_circ_0082824, hsa_circ_0068942, hsa_circ_0057576, hsa_circ_0054537, hsa_circ_0051172, hsa_circ_0032970, and hsa_circ_0006323, which sponge hsa-miR-130a$3 p$ that regulates TRPM3. This suggests that the cohort of circRNAs negatively regulate miR-130A-3p, thereby resulting in upregulation of TRPM3.

This study has several limitations that need to be addressed while interpreting our results. First, the ceRNA

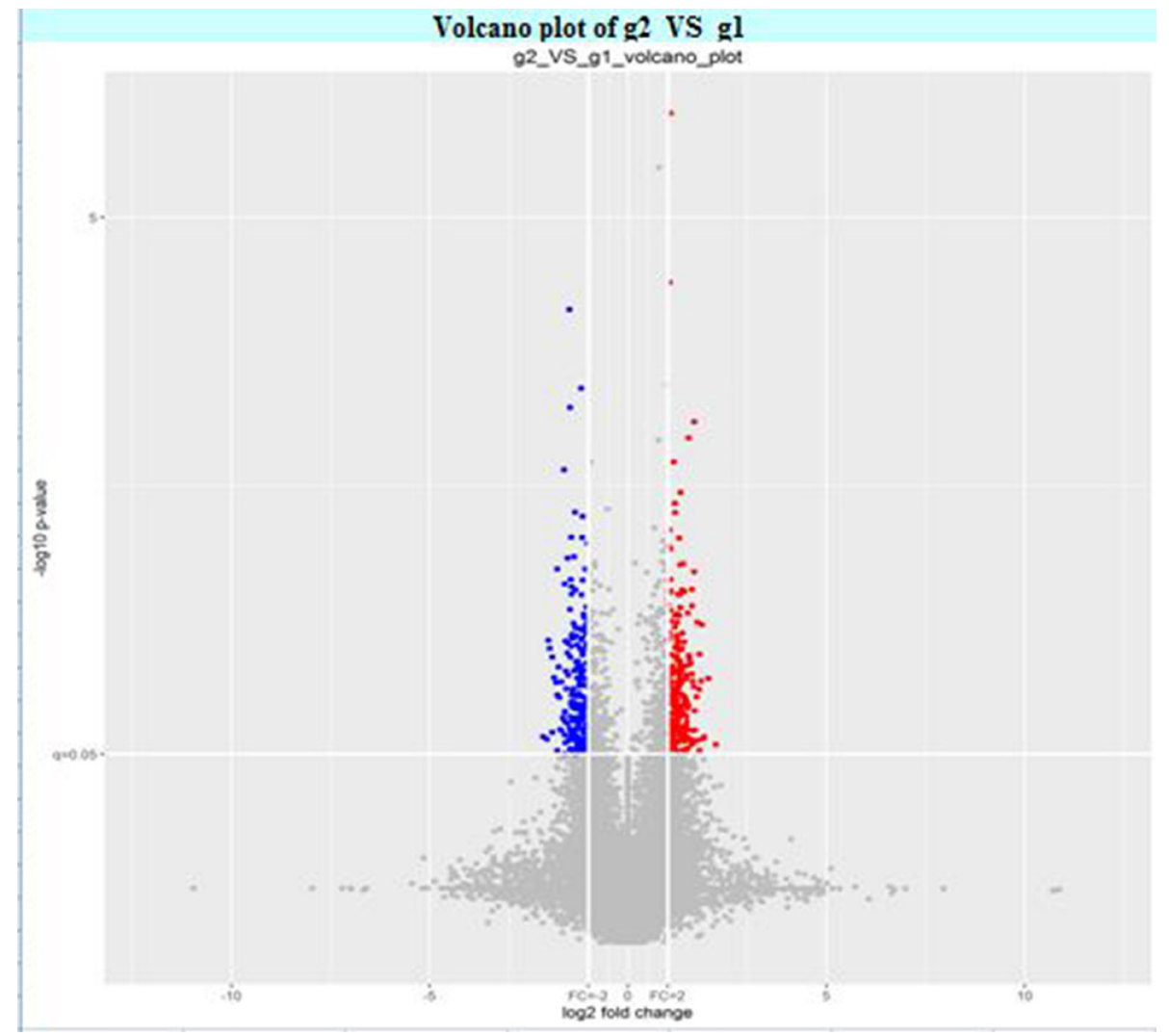

Figure 2: Volcano plot of the differentially expressed plasma circRNAs in CAD and control subjects. 
Table 3: Differentially expressed miRNAs in the 2nd set of CAD patients

\begin{tabular}{ccccc}
\hline Characteristics & $\begin{array}{c}\text { CADs } \\
(\boldsymbol{n}=\mathbf{6 4 8})\end{array}$ & $\begin{array}{c}\text { Controls } \\
(\boldsymbol{n}=\mathbf{2 8 4})\end{array}$ & Mann-Whitney U & $\boldsymbol{P}$ value \\
\hline miR-125b & $0.03(0.00-0.23)$ & $0.04(0.00-0.27)$ & 90214.00 & 0.615 \\
miR-122 & $0.17(0.00-1.82)$ & $0.29(0.01-1.76)$ & 87021.50 & 0.182 \\
miR-214 & $0.00(0.00-0.24)$ & $0.01(0.00-0.26)$ & 88581.50 & 0.333 \\
miR-133b & $0.15(0.00-0.45)$ & $0.19(0.00-0.44)$ & 89564.00 & 0.504 \\
miR-221 & $0.09(0.03-0.17)$ & $0.12(0.05-0.26)$ & 79349.00 & 0.001 \\
miR-21 & $1.95(0.60-4.76)$ & $1.69(0.64-4.20)$ & 91569.00 & 0.906 \\
miR-155 & $151.17(43.41-2557.31)$ & $246.43(65.91-2812.24)$ & 84587.50 & 0.049 \\
miR-106a & $1.48(0.74-2.98)$ & $1.62(0.87-3.13)$ & 85901.50 & 0.106 \\
miR-130a & $2.97(1.44-5.24)$ & $3.48(1.71-11.57)$ & 79968.00 & 0.001 \\
\hline
\end{tabular}

CAD, coronary artery disease. The value of each miRNAs means the relative mount calculated by $2^{-\Delta c t}$ method.

microarray was based on a small group of $3 \mathrm{CAD}$ and 3 control subjects. Since the expression of circRNA and ceRNA can vary in individuals due to a number of factors, the circRNA and mRNA profiles were verified in another independent cohort of 932 subjects by qRT-PCR. The in vivo relevance of our findings requires further comprehensive investigation. Second, the mechanism of circRNA regulation of hsa-miR-130a-3p and TRPM3 is based on the bioinformatics analysis and needs to be tested in in vitro and in vivo models.

In conclusion, we identified a network of 9 circRNAs that regulate TRPM3 expression by inhibiting hsa-miR-130a-3p in CAD patients.

\section{MATERIALS AND METHODS}

\section{Study subjects}

In the first group, we enrolled $3 \mathrm{CAD}$ and 3 control (4 males and 2 females) subjects at the Yancheng TCM Hospital Affiliated to Nanjing University of Chinese Medicine in China. The study was performed as approved by the ethics committee of the Yancheng TCM Hospital Affiliated to Nanjing University of Chinese Medicine and the First Affiliated Hospital of Nanjing Medical University. All subjects provided written informed consent. The characteristics of the study subjects are shown in Table 6 .

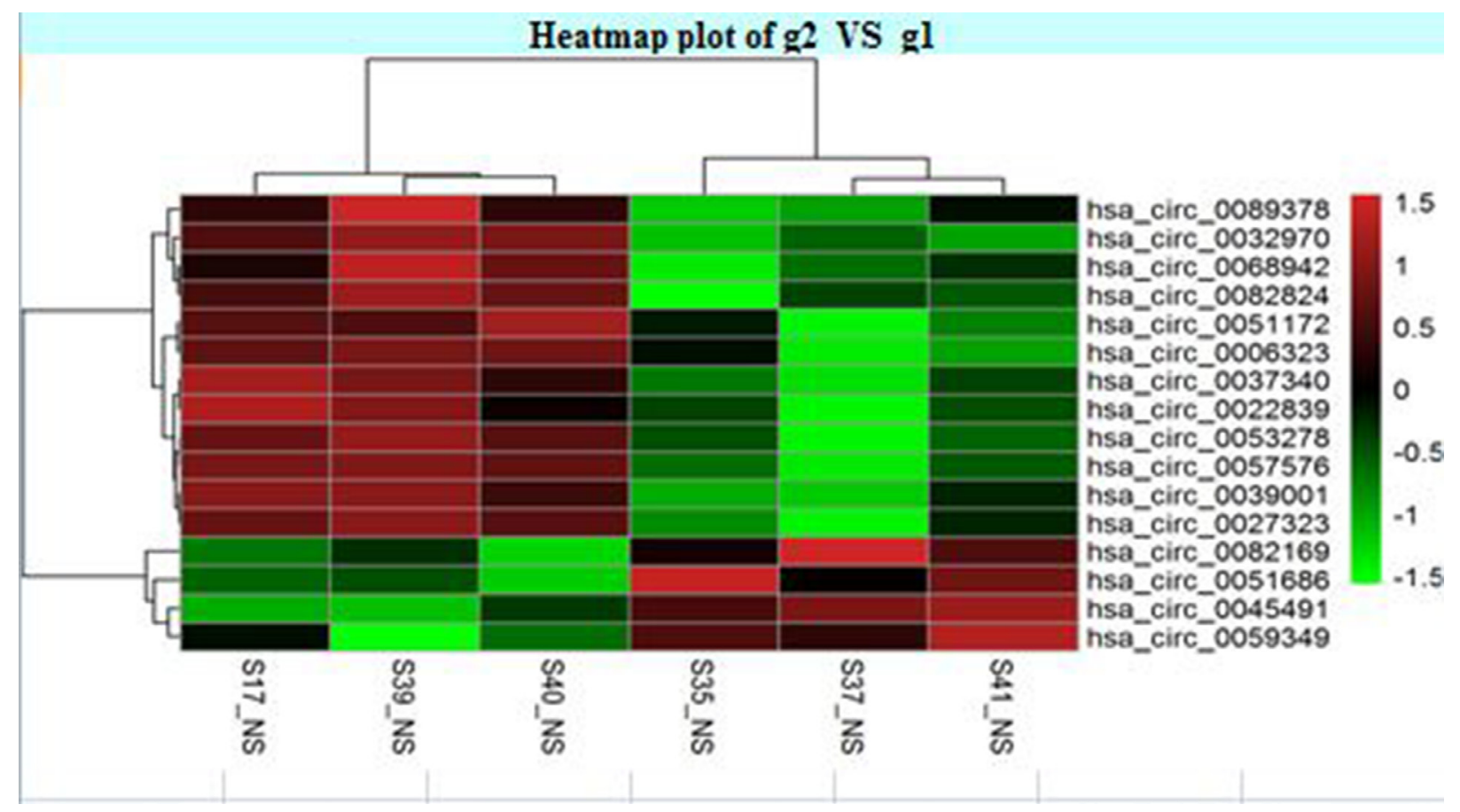

Figure 3: Plasma circRNA profile of CAD and control subjects. Heat map shows the cirRNAs with $>1.5$ fold changes. The cirRNAs are hierarchically clustered on the y-axis based on their expression. The expression index is color coded with green indicating downregulation and red indicating upregulation. 
Table 4: MicroRNA-circRNA interactions in CAD

\begin{tabular}{|c|c|c|c|c|}
\hline NO. & miRNA & $\begin{array}{c}\text { miRNAup/down } \\
\text { regulation }\end{array}$ & $\operatorname{circRNA}$ & $\begin{array}{c}\text { circRNA up/down } \\
\text { regulation }\end{array}$ \\
\hline 1 & hsa-miR-221-3p & down & hsa_circ_0039001 & up \\
\hline 2 & hsa-miR-221-3p & down & hsa_circ_0083357 & up \\
\hline 3 & hsa-miR-221-3p & down & hsa_circ_0038998 & up \\
\hline 4 & hsa-miR-221-3p & down & hsa_circ_0089378 & up \\
\hline 5 & hsa-miR-221-3p & down & hsa_circ_0032970 & up \\
\hline 6 & hsa-miR-221-3p & down & hsa_circ_0068942 & up \\
\hline 7 & hsa-miR-221-3p & down & hsa_circ_0051172 & up \\
\hline 8 & hsa-miR-221-3p & down & hsa_circ_0054537 & up \\
\hline 9 & hsa-miR-221-3p & down & hsa_circ_0006323 & up \\
\hline 10 & hsa-miR-221-3p & down & hsa_circ_0082824 & up \\
\hline 11 & hsa-miR-155-5p & down & hsa_circ_0026666 & up \\
\hline 12 & hsa-miR-155-5p & down & hsa_circ_0083357 & up \\
\hline 13 & hsa-miR-155-5p & down & hsa_circ_0038998 & up \\
\hline 14 & hsa-miR-155-5p & down & hsa_circ_0089378 & up \\
\hline 15 & hsa-miR-155-5p & down & hsa_circ_0032970 & up \\
\hline 16 & hsa-miR-155-5p & down & hsa_circ_0068942 & up \\
\hline 17 & hsa-miR-155-5p & down & hsa_circ_0051172 & up \\
\hline 18 & hsa-miR-155-5p & down & hsa_circ_0054537 & up \\
\hline 19 & hsa-miR-155-5p & down & hsa_circ_0006323 & up \\
\hline 20 & hsa-miR-155-5p & down & hsa_circ_0082824 & up \\
\hline 21 & hsa-miR-155-5p & down & hsa_circ_0053278 & up \\
\hline 22 & hsa-miR-155-5p & down & hsa_circ_0057576 & up \\
\hline 23 & hsa-miR-130a-3p & down & hsa_circ_0083357 & up \\
\hline 24 & hsa-miR-130a-3p & down & hsa_circ_0089378 & up \\
\hline 25 & hsa-miR-130a-3p & down & hsa_circ_0032970 & up \\
\hline 26 & hsa-miR-130a-3p & down & hsa_circ_0068942 & up \\
\hline 27 & hsa-miR-130a-3p & down & hsa_circ_0051172 & up \\
\hline 28 & hsa-miR-130a-3p & down & hsa_circ_0054537 & up \\
\hline 29 & hsa-miR-130a-3p & down & hsa_circ_0006323 & up \\
\hline 30 & hsa-miR-130a-3p & down & hsa_circ_0082824 & up \\
\hline 31 & hsa-miR-130a-3p & down & hsa_circ_0057576 & up \\
\hline
\end{tabular}

Table 5: Hsa-miR-130a-3p-mediated circRNA-mRNA ceRNA network

\begin{tabular}{cccc}
\hline Name & up/down regulation & Degree & ceRNA type \\
\hline hsa-miR-130a-3p & down & 10 & mRNA \\
TRPM3 & up & 1 & CircRNA \\
hsa_circ_0089378 & up & 1 & CircRNA \\
hsa_circ_0083357 & up & 1 & CircRNA \\
hsa_circ_0082824 & up & 1 & CircRNA \\
hsa_circ_0068942 & up & 1 & CircRNA \\
hsa_circ_0057576 & up & 1 & CircRNA \\
hsa_circ_0054537 & up & 1 & CircRNA \\
hsa_circ_0051172 & up & 1 & CircRNA \\
hsa_circ_0032970 & up & 1 & CircRNA \\
hsa_circ_0006323 & up & 1 & \\
\hline
\end{tabular}


In the second group, 932 consecutive adult subjects (681 males and 251 females, 648 CAD subjects and 284 controls) aged 32-84 years that underwent coronary angiography for suspected or known coronary atherosclerosis was analyzed. They were part of a previous study on CAD. Subjects with spastic angina pectoris, infectious processes within 2 weeks, heart failure, adrenal dysfunction, and thyroid dysfunction were excluded from this study. The plasma miRNA levels were confirmed by qRT-PCR analysis. Circulating levels of miRNAs were quantified using the $2^{-\Delta \mathrm{ct}}$ method.

\section{Microarray analysis}

Total RNA was extracted from the plasma of the subjects and purified using the mirVana ${ }^{\mathrm{TM}}$ PARIS $^{\mathrm{TM}}$ kit (Cat\#AM1556, Ambion, Austin, TX, USA) according to the manufacturer's instructions. The RNA integration number (RIN) was determined by an Agilent Bioanalyzer 2100 (Agilent technologies, Santa Clara, CA, USA). Total RNA $(1 \mu \mathrm{g})$ was amplified and labeled by Low Input Quick Amp WT Labeling Kit (Cat. \# 5190-2943, Agilent technologies, Santa Clara, CA, USA) according to the manufacturer's instructions. Labeled cRNA were purified by RNeasy mini kit (Cat.\# 74106, QIAGEN, GmBH, Germany).

The cDNA was labeled and hybridized to the human SBC-ceRNA (4x180k) Array, which can detect
88,371 circRNAs, and 18,853 coding transcripts (Circbase (88371), GENCODE v21/Ensembl (18,100), LNCipedia v3.1 (40,621), Lncrnadb (28), Noncode v4 $(2,608)$, UCSC $(25,919)$ databases $)$. We hybridized $1 \mu \mathrm{g}$ Cy3-labeled cRNA onto each slide using Gene Expression Hybridization Kit (Cat.\# 5188-5242, Agilent technologies, Santa Clara, CA, US) in an hybridization oven (Cat.\# G2545A, Agilent technologies, Santa Clara, CA, US) according to the manufacturer's instructions for $17 \mathrm{~h}$. Then, slides were washed in staining dishes (Cat.\# 121, Thermo Shandon, Waltham, MA, USA) with Gene Expression Wash Buffer Kit (Cat.\# 5188-5327, Agilent technologies, Santa Clara, CA, USA) according to the manufacturer's instructions.

The slides were scanned by Agilent Microarray Scanner (Cat\#G2565CA, Agilent technologies, Santa Clara, CA, USA) with default settings (Dye channel: Green; Scan resolution $=3 \mu \mathrm{m}$; PMT 100\%; 20 bit). Raw data was extracted with Feature Extraction software 10.7 (Agilent technologies, Santa Clara, CA, USA) and normalized by Quantile algorithm and LIMMA packages in $R$. The microarray work was performed by Shanghai Biotechnology Cooperation, Shanghai, P.R. China.

\section{Construction of ceRNA network}

Figure 5 shows the methodology used to identify the ceRNA interacting genes. The miRanda method, which

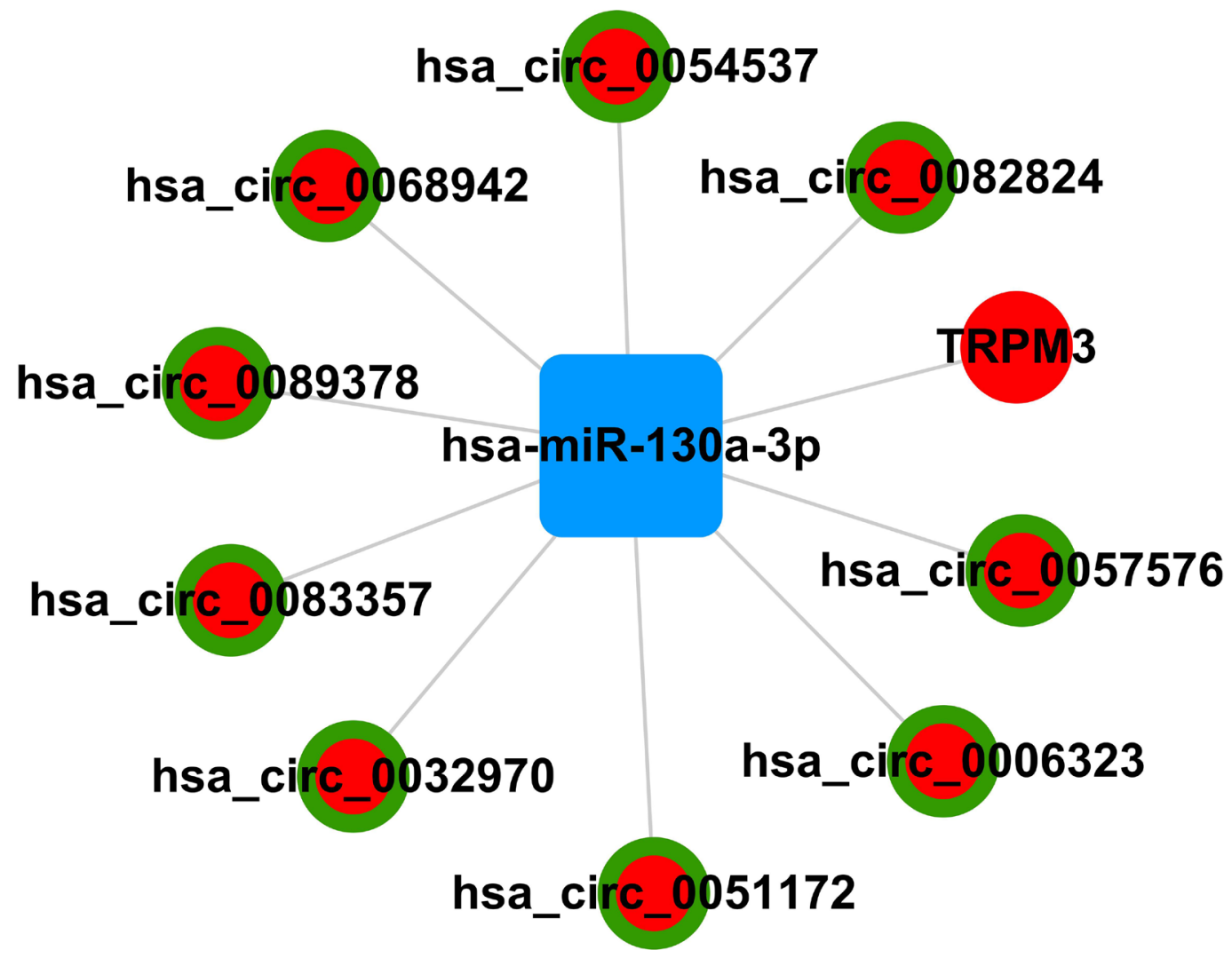

Figure 4: Schematic representation of hsa-miR-130a-3p-mediated circRNA-mRNA ceRNA network. 
Table 6: Characteristics of the CAD study population

\begin{tabular}{|c|c|c|c|}
\hline Characteristics & $\begin{array}{l}\text { CAD } \\
(N=3)\end{array}$ & $\begin{array}{l}\text { Control } \\
(N=3)\end{array}$ & Total \\
\hline Age (years) & $64.67 \pm 10.02$ & $49.00 \pm 2.65$ & $56.83 \pm 10.80$ \\
\hline Sex (male/female) & $2 / 1$ & $2 / 1$ & $4 / 2$ \\
\hline \multicolumn{4}{|l|}{ Physical data } \\
\hline Heart rate (bpm.) & $71.33 \pm 1.15$ & $80.00 \pm 20.00$ & $75.67 \pm 13.53$ \\
\hline Height $(\mathrm{cm})$ & $160 \pm 7$ & $167 \pm 7$ & $164 \pm 7$ \\
\hline $\operatorname{BMI}\left(\mathrm{kg} / \mathrm{m}^{2}\right)$ & $26.97 \pm 3.41$ & $24.60 \pm 4.18$ & $25.55 \pm 3.65$ \\
\hline \multicolumn{4}{|l|}{ Historical data } \\
\hline Diabetes mellitus (Y/N) & $0 / 3$ & $0 / 3$ & $0 / 6$ \\
\hline Arterial hypertension & $3 / 0$ & $0 / 3$ & $3 / 3$ \\
\hline Dyslipidaemia $(\mathrm{Y} / \mathrm{N})$ & $3 / 0$ & $1 / 2$ & $4 / 2$ \\
\hline Family history (Y/N) & $0 / 3$ & $0 / 3$ & $0 / 6$ \\
\hline \multicolumn{4}{|l|}{ Laboratory data } \\
\hline Glucose (mM) & $5.45 \pm 0.28$ & $6.48 \pm 2.53$ & $5.97 \pm 1.71$ \\
\hline TC (mM) & $4.58 \pm 0.39$ & $4.10 \pm 0.61$ & $4.34 \pm 0.53$ \\
\hline TG (mM) & $1.68 \pm 0.51$ & $1.77 \pm 0.26$ & $1.73 \pm 0.37$ \\
\hline HDL (mM) & $1.00 \pm 0.26$ & $0.80 \pm 0.44$ & $0.90 \pm 0.34$ \\
\hline LDL (mM) & $2.85 \pm 0.46$ & $2.46 \pm 0.72$ & $2.66 \pm 0.58$ \\
\hline ApoA1 (g/L) & $1.12 \pm 0.18$ & $1.00 \pm 0.22$ & $1.06 \pm 0.19$ \\
\hline ApoB (g/L) & $0.95 \pm 0.08$ & $0.85 \pm 0.25$ & $0.90 \pm 1.18$ \\
\hline $\mathrm{T}-\mathrm{Bil}(\mu \mathrm{M})$ & $24.33 \pm 14.09$ & $13.83 \pm 1.85$ & $19.08 \pm 10.67$ \\
\hline D-Bil $(\mu \mathrm{M})$ & $6.53 \pm 3.70$ & $4.20 \pm 0.70$ & $5.37 \pm 2.70$ \\
\hline Total protein $(\mathrm{g} / \mathrm{L})$ & $67.23 \pm 3.26$ & $66.00 \pm 5.02$ & $66.62 \pm 3.85$ \\
\hline Albumin $(\mathrm{g} / \mathrm{L})$ & $40.27 \pm 1.38$ & $40.50 \pm 0.72$ & $40.38 \pm 0.99$ \\
\hline Sodium (mM) & $140.63 \pm 1.72$ & $141.30 \pm 1.06$ & $140.97 \pm 1.33$ \\
\hline Potassium (mM) & $3.37 \pm 0.39$ & $3.95 \pm 0.29$ & $3.66 \pm 0.44$ \\
\hline Chloride (mM) & $103.77 \pm 1.40$ & $105.03 \pm 0.58$ & $104.40 \pm 1.18$ \\
\hline Calcium (mM) & $2.25 \pm 0.09$ & $2.28 \pm 0.08$ & $2.27 \pm 0.07$ \\
\hline Urea (mM) & $5.87 \pm 1.61$ & $4.48 \pm 0.76$ & $5.18 \pm 1.36$ \\
\hline Uric acid $(\mu \mathrm{M})$ & $272.93 \pm 56.42$ & $385.57 \pm 68.48$ & $329.25 \pm 83.40$ \\
\hline $\mathrm{RBC}\left(10^{12} / \mathrm{L}\right)$ & $4.58 \pm 0.40$ & $4.79 \pm 0.57$ & $4.69 \pm 0.45$ \\
\hline WBC $\left(10^{9} / \mathrm{L}\right)$ & $8.31 \pm 2.75$ & $5.95 \pm 2.10$ & $7.13 \pm 2.54$ \\
\hline $\operatorname{PLT}\left(10^{9} / \mathrm{L}\right)$ & $135.67 \pm 40.50$ & $170.33 \pm 92.09$ & $153.00 \pm 66.40$ \\
\hline HGB (g/L) & $144.33 \pm 6.51$ & $143.33 \pm 18.50$ & $143.83 \pm 12.42$ \\
\hline \multicolumn{4}{|l|}{ Smoking status } \\
\hline Current (Y/N) & $1 / 2$ & $1 / 2$ & $2 / 4$ \\
\hline Former $(\mathrm{Y} / \mathrm{N})$ & $0 / 3$ & $0 / 3$ & $0 / 6$ \\
\hline Never $(\mathrm{Y} / \mathrm{N})$ & $2 / 1$ & $2 / 1$ & $4 / 2$ \\
\hline \multicolumn{4}{|c|}{ Major epicardial vessel with $>\mathbf{5 0 \%}$ stenosis } \\
\hline $\mathrm{LAD}(\mathrm{Y} / \mathrm{N})$ & $3 / 0$ & $0 / 3$ & $3 / 3$ \\
\hline $\mathrm{LCX}(\mathrm{Y} / \mathrm{N})$ & $0 / 3$ & $0 / 3$ & $0 / 6$ \\
\hline $\mathrm{RCA}(\mathrm{Y} / \mathrm{N})$ & $0 / 3$ & $0 / 3$ & $0 / 6$ \\
\hline
\end{tabular}




\section{Treatment}

\begin{tabular}{llll} 
ACE-I (Y/N) & $0 / 3$ & $0 / 3$ & $0 / 6$ \\
ARB (Y/N) & $2 / 1$ & $0 / 3$ & $2 / 4$ \\
Beta-blocker (Y/N) & $1 / 2$ & $1 / 2$ & $2 / 4$ \\
CCB (Y/N) & $2 / 1$ & $0 / 3$ & $2 / 4$ \\
Diuretics (Y/N) & $0 / 3$ & $0 / 3$ & $0 / 6$ \\
Statins (Y/N) & $3 / 0$ & $2 / 1$ & $5 / 1$ \\
Anti-platelet therapy (Y/N) & $3 / 0$ & $3 / 0$ & $6 / 0$ \\
\hline
\end{tabular}

Data are presented as mean + SD. n, numbers of patients.

CAD, coronary artery heart disease; BMI, body mass index $\left(\mathrm{kg} / \mathrm{m}^{2}\right)$; TC, total cholesterol; TG, triglyceride; HDL, highdensity lipoprotein; LDL, low-density lipoprotein; ApoA1, apolipoprotein A1; ApoB, apolipoprotein B; T-Bil, total bilirubin; D-Bil, direct bilirubin; RBC, red blood cell; WBC, white blood cell; PLT, platelet; HGB, haemoglobin; LAD, left anterior descending; LCX, left circumflex; RCA, right coronary artery; ACEI, angiotensin-converting enzyme inhibitor; ARB, angiotensin receptor blocker; $\mathrm{CCB}$, calcium channel blocker.

is based on dynamic programming ( $\mathrm{SW}$ algorithm) and computing free energy was used to identify the target genes of miRNAs and ceRNAs [21-25]. Based on this analysis, we built a microRNA-cirRNA-mRNA interaction network. The relationship between the target and the microRNA was based on the adjacency matrix of microRNA and target A $=[a i, j]$, where ai,j represents the weight of the relationship between the target (i) and its microRNA (j). In the microRNA-cirRNA-mRNA network, the circle represents one edge, whereas the center of the network represents a degree. The degree denotes the contribution of a microRNA to the target gene around it or the contribution of a target to the microRNAs around it. The key microRNAs and their targets in the network always had the largest degrees.

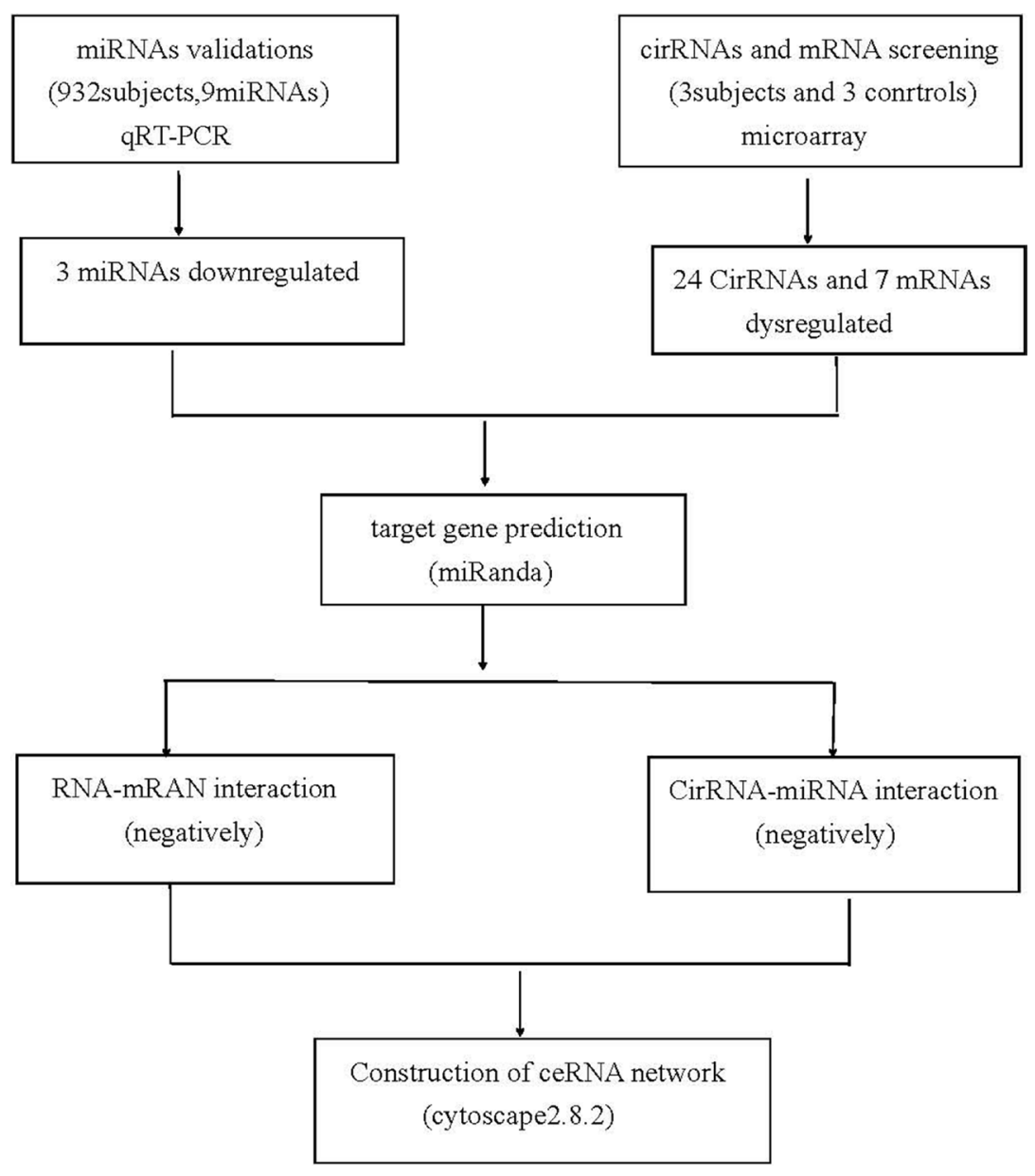

Figure 5: Flow chart of CAD related competing endogenous RNA network study. 


\section{ACKNOWLEDGMENTS AND FUNDING}

This study was supported by grants from the National Natural Science Foundations of China (grant numbers 81170180, 30400173 and 30971257) and the Priority Academic Program Development of Jiangsu Higher Education Institutions.

\section{CONFLICTS OF INTEREST}

The authors declare that no conflicts of interest exist.

\section{REFERENCES}

1. WHO. Global Status Report on noncommunicable diseases 2014. Available at: http://www.thehealthwell.info/ node/831799 Accessed: December 18, 2015.

2. WHO. Cardiovascular diseases mortality: age-standardized death rate per 100,000 population, 2000-2012. Available at: http://gamapserver.who.int/gho/interactive_charts/ncd/ mortality/cvd/atlas.html Accessed: December 18, 2015.

3. Mozaffarian D, Benjamin EJ, Go AS, Arnett DK, Blaha MJ, Cushman M, de Ferranti S, Després JP, Fullerton HJ, Howard VJ, Huffman MD, Judd SE, Kissela BM, et al. Heart disease and stroke statistics - 2015 update: a report from the American Heart Association. Circulation. 2015; 131:e29-322.

4. Leal J, Luengo-Fernandez R, Gray A, Rayner M. Economic burden of cardiovascular diseases in the enlarged European Union. Eur Heart J. 2006; 27:1610-9.

5. Pennisi E. Genomics. ENCODE project writes eulogy for junk DNA. Science. 2012; 337:1159-61.

6. Liu G, Mattick JS, Taft RJ. A meta-analysis of the genomic and transcriptomic composition of complex life. Cell Cycle. $2013 ; 12: 2061-72$.

7. Arner E, Daub CO, Vitting-Seerup K, Andersson R, Lilje B, Drabløs F, Lennartsson A, Rönnerblad M, Hrydziuszko O, Vitezic M, Freeman TC, Alhendi AM, Arner P, et al. Transcribed enhancers lead waves of coordinated transcription in transitioning mammalian cells. Science. 2015; 347:1010-4

8. Schlosser K, Hanson J, Villeneuve PJ, Dimitroulakos J, McIntyre L, Pilote L, Stewart DJ. Assessment of Circulating LncRNAs Under Physiologic and Pathologic Conditions in Humans Reveals Potential Limitations as Biomarkers. Sci Rep. 2016; 6:36596. https://doi.org/10.1038/srep36596.

9. Yang Y, Cai Y, Wu G, Chen X, Liu Y, Wang X, Yu J, Li C, Chen X, Jose PA, Zhou L, Zeng C. Plasma long non-coding RNA, CoroMarker, a novel biomarker for diagnosis of coronary artery disease. Clin Sci (Lond). 2015; 129:675-85.

10. Chen J, Li Y, Zheng Q, Bao C, He J, Chen B, Lyu D, Zheng B, $\mathrm{Xu}$ Y, Long Z, Zhou Y, Zhu H, Wang Y, et al. Circular RNA profile identifies circPVT1 as a proliferative factor and prognostic marker in gastric cancer. Cancer Lett. 2016; pii:S0304-3835(16)30751-0.

11. Wang K, Long B, Liu F, Wang JX, Liu CY, Zhao B, Zhou LY, Sun T, Wang M, Yu T, Gong Y, Liu J, Dong YH, et al. A circular RNA protects the heart from pathological hypertrophy and heart failure by targeting miR-223. Eur Heart J. 2016; 37:2602-11. https://doi.org/10.1093/ eurheartj/ehv713.

12. Song C, Zhang J, Liu Y, Pan H, Qi HP, Cao YG, Zhao JM, Li S, Guo J, Sun HL, Li CQ. Construction and analysis of cardiac hypertrophy-associated lncRNA-mRNA network based on competitive endogenous RNA reveal functional lncRNAs in cardiac hypertrophy. Oncotarget. 2016; 7:10827-40. https://doi.org/10.18632.oncotarget.7312.

13. Jia QW, Chen ZH, Ding XQ, Liu JY, Ge PC, An FH, Li LH, Wang LS, Ma WZ, Yang ZJ, Jia EZ. Predictive Effects of Circulating miR-221, miR-130a and miR-155 for Coronary Heart Disease: A Multi-Ethnic Study in China. Cell Physiol Biochem. 2017; 42:808-823.

14. Pang L, Hu J, Zhang G, Li X, Zhang X, Yu F, Lan Y, Xu J, Pang B, Han D, Xiao Y, Li X. Dysregulated long intergenic non-coding RNA modules contribute to heart failure. Oncotarget. 2016; 7:59676-59690. https://doi. org/10.18632/oncotarget.10834.

15. Ling S, Birnbaum Y, Nanhwan MK, Thomas B, Bajaj M, Li Y, Li Y, Ye Y. Dickkopf-1 (DKK1) phosphatase and tensin homolog on chromosome 10 (PTEN) crosstalk via microRNA interference in the diabetic heart. Basic Res Cardiol. 2013; 108:352. https://doi.org/10.1007/s00395-013-0352-2.

16. de Gonzalo-Calvo D, Cenarro A, Garlaschelli K, Pellegatta F, Vilades D, Nasarre L, Camino-Lopez S, Crespo J, Carreras F, Leta R, Catapano AL, Norata GD, Civeira F, et al. Translating the microRNA signature of microvesicles derived from human coronary artery smooth muscle cells in patients with familial hypercholesterolemia and coronary artery disease. J Mol Cell Cardiol. 2017; 106:55-67. https:// doi. org/10.1016/j.yjmcc.2017.03.005.

17. Staszel T, Zapała B, Polus A, Sadakierska-Chudy A, Kieć-Wilk B, Stępień E, Wybrańska I, Chojnacka M, Dembińska-Kieć A. Role of microRNAs in endothelial cell pathophysiology. Pol Arch Med Wewn. 2011; 121:361-6

18. Zhang Q, Kandic I, Kutryk MJ. Dysregulation of angiogenesis-related microRNAs in endothelial progenitor cells from patients with coronary artery disease. Biochem Biophys Res Commun. 2011; 405:42-6. https://doi. org/10.1016/j.bbrc.2010.12.119.

19. Naylor J, Li J, Milligan CJ, Zeng F, Sukumar P, Hou B, Sedo A, Yuldasheva N, Majeed Y, Beri D, Jiang S, Seymour VA, McKeown L, et al. Pregnenolone sulphateand cholesterol-regulated TRPM3 channels coupled to vascular smooth muscle secretion and contraction. Circ Res. 2010; 106:1507-15.

20. Zhou B, Xu HY. A novel identified circular RNA, circRNA_010567, promotes myocardial fibrosis via suppressing miR-141 by targeting TGF- $\beta 1$. Biochem Biophys Res Commun. 2017; S0006-291X(17)30710-6. https://doi.org/10.1016/j.bbrc.2017.04.044.

21. Lewis BP, Burge CB, Bartel DP. Conserved Seed Pairing, Often Flanked by Adenosines, Indicates that Thousands of Human Genes are MicroRNA Targets. Cell. 2005; 120:15-20. 
22. Friedman RC, Farh KK, Burge CB, Bartel DP. Most Mammalian mRNAs Are Conserved Targets of MicroRNAs. Genome Research. 2009; 19:92-105.

23. Grimson A, Farh KK, Johnston WK, Garrett-Engele P, Lim LP, Bartel DP. MicroRNA Targeting Specificity in Mammals: Determinants beyond Seed Pairing. Molecular Cell. 2007; 27:91-105.
24. Garcia DM, Baek D, Shin C, Bell GW, Grimson A, Bartel DP. Weak Seed-Pairing Stability and High Target-Site Abundance Decrease the Proficiency of 1sy- 6 and Other miRNAs. Nat Struct Mol Biol. 2011; 18:1139-1146.

25. Myers EW, Miller W. Approximate matching of regular expressions. Bull Math Biol. 1989; 51:5-37. 